\title{
Resilience in Diet-quality Strategies of Mixed Native and Non-native Herbivore Communities in Semi-arid and Sub-humid Savanna Ecosystem
}

\section{Stoklasová Lucie}

Czech University of Life Sciences Prague

Pavla Hejcmanová ( $\square$ hejcmanova@ftz.czu.cz)

Czech University of Life Sciences Prague

\section{Research Article}

Keywords: animal foraging, diet quality, herbivore feeding guilds, functional diversity, lignin, macroelements, resilience, savanna, West Africa

Posted Date: March 6th, 2021

DOI: https://doi.org/10.21203/rs.3.rs-282862/v1

License: (c) (i) This work is licensed under a Creative Commons Attribution 4.0 International License.

Read Full License 


\section{Abstract}

The global climate change processes are expected to impact African savanna ecosystems in their ecological functionality and availability of food resources for herbivores. To understand the feeding responses of large savanna herbivores placed to space-constrained and two environmentally distinct (semi-arid versus sub-humid savanna) conditions, we investigated the diet quality of five species living in a mixed community of species native and non-native for the West African savanna and compared them between the two sites as a proxy for a potential ecosystem shift due to climate change. Grazers and mixed feeders maintained diet quality in most nutrients at similar levels, while browser's diet had lower nitrogen and fibres, specifically lignin, and more calcium in semi-arid savanna. Our findings suggest that adaptation to different ecosystems with no possibility to leave the area required changes in feeding behaviour across feeding types to maintain diet quality, especially mixed feeders escaped the competition with zebras for grasses in sub-humid savanna by switching to browse. Pure browser experienced reduced diet quality in drier environment and may potentially become susceptible to ecosystem changes. Conservation strategies should facilitate both, animals' adequate behavioural responses together with nutritional resilience in changing savanna landscapes.

\section{Introduction}

Dietary choices of large mammalian herbivores are complex and animals use a wide variety of foraging strategies, based basically on their evolutionary adaptations and ecological processes, i.e. competition, facilitation, and habitat selection, to acquire and utilize foodstuffs $[1 ; 2]$. While the diets are consistent at the plant functional level within feeding guilds [2;3], there is evidence on dietary richness at plant-species levels within guilds which underpins their coexistence, particularly in African savannas $[4 ; 2 ; 5]$. The seasonal character of African savanna climate plays the primer role in these processes, specifically shaping the competition forces to strengthen during the dry season when the high-quality resources are scarce [6; 7]. Coexistence of herbivores is therefore driven by resource partitioning, which enables species consuming the same source of food to acquire sufficient amount of forage for survival [5]. Among native herbivores, overlap in resource use is not expected due to evolutionary segregation. In a native assemblage to which an exotic species has been introduced, however, overlap in resource use can occur under food-limited conditions and consequently implies competition [7]. This is case of many wildlife reserves, where non-native species are introduced into delimited fenced areas. Animals, moreover, have to face also to the global climate change processes which are expected to increase incidence and severity of droughts [8;9] and to significantly impact availability of food resources for herbivores [10; 11]. When climate changes trigger changes in habitats, it activates animal behaviours that secure the adequate nutrition through a mechanism of resilience which may involve either shift in amount and selection of available forage or physiological processes. Animal communities thus become vulnerable as on islands surrounded by a fence, similarly as may happen to protected areas isolated in the anthropogenic matrix. The competition for changing resources may become critical and may lead to population declines and extinctions $[11 ; 12 ; 13]$. Specifically, in areas constrained by real or virtual barriers, the competition for 
limited resources and dietary partitioning represents crucial mechanisms determining the decisions for effective conservation management of large herbivore communities' diversity. The understanding of resilience of herbivore communities in fragmented landscapes to changing climatic patterns are therefore of vital concern for conservation.

To understand the feeding responses of large savanna herbivores placed to space-constrained and two environmentally distinct (semi-arid versus sub-humid savanna) conditions, we investigated the diet quality of five species living in a mixed community of species native and non-native for the West African savanna and compared them between the two sites as a proxy for a potential ecosystem shift due to climate change. The species were namely the Western Derby eland (native browser), common eland (nonnative mixed feeder/browser), roan antelope (native mixed feeder), buffalo (native grazer) and zebra (non-native grazer). We predicted that maintaining the diet quality at nutrient level represents for large herbivores a significant adaptation mechanism in satisfying species-specific nutritional requirements in divergent habitats. We examined specifically whether herbivores of different feeding guilds demonstrate distinct or the same diet quality in semi-arid and sub-humid savannas, which implicitly differ in plant species composition and abundance. Namely, we tested the differences in fibre fractions, macro- and microelements among five large ungulates belonging to grazers, mixed feeders, and browsers and then we compared their diet quality in these two savanna types in wet, dry, and late dry seasons of the year. The both sites were fenced private managed nature reserves where animals receive food supplement in the late dry season to ensure the adequate animal nutrition and to prevent animal losses. We expected therefore to depict the contribution of food supplement in the overall quality of diet during the period of most limited resources. The ultimate aim was to provide a critical missing information for the conservation of mammalian herbivores in face of potential habitat changes resulting from global climate change.

\section{Methods}

\section{Study sites}

Study was conducted in two fenced wildlife reserves in Senegal, Bandia and Fathala reserves, managed by private operators for habitat and wildlife conservation and safari-tourism. The Bandia reserve $\left(14^{\circ} 35^{\prime} \mathrm{N}, 17^{\circ} 00^{\prime} \mathrm{W} ; 3000 \mathrm{ha}\right)$, located $65 \mathrm{~km}$ southward from Dakar, has a semi-arid hot climate with annual average temperature $26^{\circ} \mathrm{C}$ and mean precipitation $450-530 \mathrm{~mm}$ divided into wet (July - October), dry (November - April) and hot dry (May - June) seasons. Vegetation is Sahelo-Sudanese savanna dominated by baobab trees (Adansonia digitata), various species of acacia (Acacia spp., Faidherbia albida), Balanites aegyptiaca, Boscia senegalensis and Cassia obtusifolia [14]. The reserve hosts large herbivores, native to Senegal such as African buffalo (Syncerus caffer brachyceros), defassa waterbuck (Kobus ellipsiprymnus defassa), roan antelope (Hippotragus equinus koba), and Western Derby eland (Taurotragus derbianus derbianus), and non-native species which have been introduced from South Africa such as giraffe (Giraffa camelopardalis giraffa), greater kudu (Tragelaphus strepsiceros), impala 
(Aepyceros melampus), common eland (Tragelaphus oryx oryx), Burchell's zebra (Equus quagga burchelli) and white rhino (Ceratotherium simum simum).

The Fathala reserve $\left(13^{\circ} 39^{\prime} \mathrm{N}, 16^{\circ} 30^{\prime} \mathrm{W} ; 2000 \mathrm{ha}\right)$ is located $250 \mathrm{~km}$ southward from Dakar, near the northern border of the Gambia. The area has a sub-humid climate, with annual average temperature $27^{\circ} \mathrm{C}$ and mean precipitation range 770-1000 mm divided into wet (July - November), dry (December March), and hot dry (April - May) seasons. Vegetation belongs to the Sudano-Guinean savannah with dominants of Acacia macrostachya, Combretum spp., Danielia olliveri, Piliostigma thonningii, Pterocarpus erinaceus, Terminalia avicennoides, T. macroptera, and Andropogon gayanus grass [15]. There are native herbivores such as bushbuck (Tragelaphus scriptus) and warthog (Phacochoerus africanus), and several introduced wildlife species from Senegal, such as African buffalo, defassa waterbuck, roan antelope, Western Derby eland, and non-native species from South Africa, such as giraffe, white rhino, zebra, and common eland.

\section{Herbivore diet evaluation}

Five herbivore species were selected as model species for diet evaluation. Grazers were represented by buffalo [16;17] and zebras [17; 18], and browsers by Derby eland [14; 15]. Mixed feeders were represented by roan antelope which tends more to grazing $[19 ; 20]$ and by common eland which tends more to browsing $[16 ; 21 ; 22]$. To evaluate the diet quality, we sampled fresh dung three times of which each represented the specific season, i.e. in December 2011 (dry season), April 2012 (hot dry season), and August 2012 (wet season) in both reserves. Sample collection was combined with observation of animals while defecating to associate correctly the dung to species. Samples were from adult males and nonlactating females. From each reserve 4-5 samples from each species at each sampling date were taken. A total of 120 samples were collected in the Bandia reserve ( 57 for Derby eland; 14 for common eland; 17 for roan antelope; 12 for buffalo; 20 for zebra) and total of 84 samples were collected in the Fathala reserve (36 for Derby eland; 10 for common eland; 17 for roan antelope; 7 for buffalo; 14 for zebra).

Samples were oven-dried at $60^{\circ} \mathrm{C}$ for at least 72 hours, then ground and homogenized. The samples were subjected to standard nutrient analyses [23] for dry matter (DM), neutral detergent fibre (NDF, AOAC no. 2002.04), acid detergent fibre and acid detergent lignin (ADF, ADL, AOAC no. 973.18). The content of hemicellulose was calculated as the difference between NDF and ADF content; the content of cellulose was calculated as the difference between ADF and lignin, both for each sample.

The $\mathrm{N}$ concentration was determined using an automated analyser TruSpec (LECO Corporation, USA) by combustion with oxygen in an oven at $950^{\circ} \mathrm{C}$. Combustion products were mixed with oxygen and the mixture passed through an infrared $\mathrm{CO} 2$ detector and through a circuit for aliquot ratio where carbon is measured as $\mathrm{CO} 2$. Gases in the aliquot circuit were transferred into helium as a carrying gas, conducted through hot copper and converted to $\mathrm{N}$.

Concentrations of $\mathrm{K}, \mathrm{S}, \mathrm{Co}, \mathrm{P}, \mathrm{Ca}, \mathrm{Mg}$, and Na were tested by ICP-OES (IRIS Intrepid II XSP Duo, THERMO Elemental, USA), and Se was analysed by hydride generation atomic absorption spectroscopy technique 
(HG-AAS) using Analyst 100 spectrometer (Perkin Elmer, USA). For ICP-OES measurements samples were mineralized in the mixture of nitric and hydrochloric acid in 6:1 ratio using a closed microwave digestion system. Then standard ICP-OES measurements followed.

Other group of elements $\mathrm{Mn}, \mathrm{Fe}, \mathrm{Cu}, \mathrm{Zn}$ and Mo was analysed by ICP - MS. Fine-grained, dry and homogenized faecal samples were acid digested in $5 \mathrm{ml} \mathrm{HNO}_{3}$ (Analpure®, Analytika, Czech Republic) for $20 \mathrm{~min}$ at $180^{\circ} \mathrm{C}$ in closed microwave digestion system (Discover SP-D, CEM Corp., USA). Final digest was diluted by water (Milli-Q purification system; Millipore, SAS, France) till volume of $45 \mathrm{ml}$, aliquot proportion $(1 \mathrm{ml})$ was removed from this volume and diluted again by $\mathrm{H}_{2} \mathrm{O}$ to the final volume of $4 \mathrm{ml}$. In such diluted digests concentration of $\mathrm{S}, \mathrm{Mn}, \mathrm{Fe}, \mathrm{Co}, \mathrm{Mn}, \mathrm{Zn}$, Se, Mo were determined by technique of inductively coupled plasma mass spectrometry (ICP-MS, Agilent 7700x, Agilent Technologies Inc., USA).

\section{Data analyses}

Data, i.e. concentrations of all elements and fibre fractions, were first tested by Kolmogorov-Smirnov test of normality. We tested for differences in concentration of elements and fibre fractions between animal species for each reserve separately using a series of one-way ANOVAs. In case of significant differences, we applied Tukey post hoc comparison test. Then, we tested the differences in concentrations of each element and fibre fractions between the reserves separately for each animal species using a series of Student's t-tests. These analyses were performed using the TIBCO ${ }^{\circledR}$ Statistica ${ }^{\text {TM }}$ package (StatSoft, Palo Alto, CA, USA).

The multivariate constrained Redundancy Analysis (RDA) in the Canoco 5 package [24] was applied to examine the relationships among the nutrients (macro-, microelements and fibre fractions) and effects of explanatory environmental variables at each wildlife reserve separately. Explanatory environmental variables were animal species and season. The data were log-transformed and standardized during the analysis. To test the significance of our constrained ordination model, unrestricted Monte Carlo permutation test (permutations $n=999$ ) was applied. Results of the analyses were visualised in the form of ordination diagrams.

\section{Results}

Overall, mixed feeders and grazers maintained the quality of their diet in most of the principal parameters, especially in the content of nitrogen and fibres, regardless the savanna ecosystem type, while browser showed significantly lower nitrogen and fibres in the semi-arid environment in comparison to the subhumid one (Table 1). The diet of all ruminants, i.e. all species except zebra, contained consistently lower proportion of indigestible lignin and higher concentration of calcium in the semi-arid environment (Table 1). The concentrations of phosphorus in diet of buffalo, roan, and Derby eland were lower in the semi-arid environment. 
Table 1

Concentrations (mean \pm SE) of macroelements and fiber fractions in faeces of large herbivores in Bandia and Fathala reserves.

\begin{tabular}{|c|c|c|c|c|c|c|}
\hline & $\begin{array}{l}\text { Derby } \\
\text { eland }\end{array}$ & $\begin{array}{l}\text { Common } \\
\text { eland }\end{array}$ & $\begin{array}{l}\text { Roan } \\
\text { antelope }\end{array}$ & Buffalo & Zebra & $\begin{array}{l}\text { F; p- } \\
\text { value }\end{array}$ \\
\hline BANDIA & $n=57$ & $n=14$ & $n=17$ & $n=12$ & $n=20$ & \\
\hline Nitrogen $\mathrm{g} / \mathrm{kg}$ & $\begin{array}{l}21.1 \pm \\
0.39^{\mathrm{b} / \star \star \star}\end{array}$ & $\begin{array}{l}24.10 \pm \\
1.27 \mathrm{~b} / \mathrm{NS}\end{array}$ & $\begin{array}{l}21.47 \pm 1.05 \\
\mathrm{~b} / \mathrm{NS}\end{array}$ & $\begin{array}{l}16.39 \pm \\
1.28^{\mathrm{a} / \mathrm{NS}}\end{array}$ & $\begin{array}{l}17.1 \pm 1.26 \\
\text { a/NS }\end{array}$ & $\begin{array}{l}9.76 \\
<0.001\end{array}$ \\
\hline $\begin{array}{l}\text { Phosphorus } \\
\text { g/kg }\end{array}$ & $\begin{array}{l}4.5 \pm \\
0.18^{\mathrm{NS} / \text { * }}\end{array}$ & $\begin{array}{l}4.95 \pm 0.62 \\
\text { NS/NS }\end{array}$ & $\begin{array}{l}4.75 \pm 0.39 \\
\mathrm{NS} / \star \star\end{array}$ & $\begin{array}{l}4.93 \pm 0.42 \\
\mathrm{NS} / \star \star\end{array}$ & $\begin{array}{l}4.1 \pm 0.41 \\
\mathrm{NS} / \mathrm{NS}\end{array}$ & $\begin{array}{l}0.95 \\
0.43\end{array}$ \\
\hline $\begin{array}{l}\text { Potassium } \\
\mathrm{g} / \mathrm{kg}\end{array}$ & $\begin{array}{l}6.7 \pm 0.16 \\
a /{ }^{*}\end{array}$ & $\begin{array}{l}6.99 \pm 0.39 \\
\mathrm{a} / \mathrm{NS}\end{array}$ & $\begin{array}{l}6.56 \pm 0.57 \\
\mathrm{a} / \mathrm{NS}\end{array}$ & $\begin{array}{l}8.14 \pm 0.68 \\
a / N S\end{array}$ & $\begin{array}{l}11.7 \pm 0.84 \\
b / N S\end{array}$ & $\begin{array}{l}22.8 \\
<0.001\end{array}$ \\
\hline Calcium g/kg & $\begin{array}{l}40.9 \pm \\
0.78^{d / * \star \star}\end{array}$ & $\begin{array}{l}35.42 \pm \\
1.71^{\mathrm{c} / \star \star}\end{array}$ & $\begin{array}{l}23.96 \pm 2.59 \\
b / \star \star \star\end{array}$ & $\begin{array}{l}17.80 \pm \\
1.73^{\mathrm{b} / *}\end{array}$ & $\begin{array}{l}8.7 \pm 0.67 \\
\text { a/NS }\end{array}$ & $\begin{array}{l}110 \\
<0.001\end{array}$ \\
\hline $\begin{array}{l}\text { Magnesium } \\
\mathrm{g} / \mathrm{kg}\end{array}$ & $\begin{array}{l}6.7 \pm 0.35 \\
\mathrm{~b} / \mathrm{NS}\end{array}$ & $\begin{array}{l}7.49 \pm 0.87 \\
\text { b/NS }\end{array}$ & $\begin{array}{l}5.54 \pm 0.42 \\
b / N S\end{array}$ & $\begin{array}{l}6.26 \pm 0.53 \\
b / N S\end{array}$ & $\begin{array}{l}3.4 \pm 0.16 \\
a / \star \star \star\end{array}$ & $\begin{array}{l}9.01 \\
<0.001\end{array}$ \\
\hline Sulphur mg/g & $\begin{array}{l}2.2 \pm \\
0.082 \mathrm{~b} / \mathrm{NS}\end{array}$ & $\begin{array}{l}2.38 \pm \\
0.089 \mathrm{~b} / \mathrm{NS}\end{array}$ & $\underset{b / \star \star \star}{2.40} \pm 0.10$ & $\begin{array}{l}2.0 \pm 0.23 \\
\mathrm{ab} / \mathrm{NS}\end{array}$ & $\begin{array}{l}1.65 \pm 0.08 \\
\mathrm{a} / \star \star \star \star\end{array}$ & $\begin{array}{l}9.24 \\
<0.001\end{array}$ \\
\hline NDF g/kg & $\underset{b / \star \star \star}{525} \pm 8.14$ & $\begin{array}{l}499 \pm 29.21 \\
\text { ab/NS }\end{array}$ & $\begin{array}{l}492 \pm 19.88 \\
a b / N S\end{array}$ & $\begin{array}{l}426 \pm 31.21 \\
\mathrm{a} / \star \star \star^{\prime}\end{array}$ & $\begin{array}{l}535 \pm 23.53 \\
b / \star *\end{array}$ & $\begin{array}{l}4.15 \\
0.004\end{array}$ \\
\hline ADF g/kg & $\underset{c / \star \star \star}{495} \pm 7.40$ & $\begin{array}{l}448 \pm 25.32 \\
\mathrm{bc} / \mathrm{NS}\end{array}$ & $\begin{array}{l}432 \pm 17.74 \\
\text { b/NS }\end{array}$ & $\begin{array}{l}344 \pm 26.89 \\
a^{/ / \star}\end{array}$ & $\begin{array}{l}390 \pm 18.17 \\
a b / \star \star\end{array}$ & $\begin{array}{l}15.6 \\
<0.001\end{array}$ \\
\hline Cellulose & $\begin{array}{l}224 \pm 4.85 \\
\mathrm{a} / \mathrm{NS}\end{array}$ & $\begin{array}{l}213 \pm 14.26 \\
a^{/ * \star}\end{array}$ & $\begin{array}{l}237 \pm 14.03 \\
\text { ab/NS }\end{array}$ & $\begin{array}{l}202 \pm 17.82 \\
a / *\end{array}$ & $\underset{b / \star \star}{263} \pm 15.34$ & $\begin{array}{l}3.62 ; \\
0.008\end{array}$ \\
\hline Hemicellulose & $\begin{array}{l}30 \pm 2.89 \\
\mathrm{a}^{/ \star \star \star \star}\end{array}$ & $\begin{array}{l}51 \pm 10.04 \\
a b / N S\end{array}$ & $\begin{array}{l}60 \pm 9.12 \\
b / N S\end{array}$ & $\begin{array}{l}82 \pm 8.84 \\
\text { b/NS }\end{array}$ & $\begin{array}{l}145 \pm 10.49 \\
\text { c/NS }\end{array}$ & $\begin{array}{l}48.1 ; \\
<0.001\end{array}$ \\
\hline Lignin $\mathrm{g} / \mathrm{kg}$ & $\begin{array}{l}271 \pm 5.27 \\
d / \star \star \star\end{array}$ & $\begin{array}{l}235 \pm 14.13 \\
\mathrm{c} / \mathrm{NS}\end{array}$ & $\begin{array}{l}195 \pm 8.82 \\
b / N S\end{array}$ & $\begin{array}{l}142 \pm 10.34 \\
a^{/ / \star \star \star}\end{array}$ & $\begin{array}{l}128 \pm 6.24 \\
\text { a/NS }\end{array}$ & $\begin{array}{l}66.5 ; \\
<0.001\end{array}$ \\
\hline $\begin{array}{l}\text { Lignin } \\
\text { proportion \% }\end{array}$ & $\begin{array}{l}54.7^{ \pm} \\
0.6^{\mathrm{C}^{/ \star \star \star}}\end{array}$ & $\begin{array}{l}52.6 \pm 1.4 \\
\mathrm{c} / \star \star \star\end{array}$ & $\begin{array}{l}45.6 \pm 1.6 \\
b / *\end{array}$ & $\begin{array}{l}41.9 \pm 1.3 \\
b / * \star\end{array}$ & $\begin{array}{l}33.2 \pm 1.3 \\
\text { a/ NS }\end{array}$ & $\begin{array}{l}66.8 \\
<0.001\end{array}$ \\
\hline Ca:P & $\begin{array}{l}11.18 \pm \\
1.0^{\mathrm{c} / \star \star \star}\end{array}$ & $\begin{array}{l}8.1 \pm \\
0.89^{\mathrm{b} / \star \star \star}\end{array}$ & $\begin{array}{l}5.19 \pm \\
0.74^{\mathrm{ab} / \mathrm{NS}}\end{array}$ & $\begin{array}{l}3.5 \pm \\
0.34^{\mathrm{a} / * \star}\end{array}$ & $\begin{array}{l}2.7 \pm \\
0.38^{\mathrm{a} / \mathrm{NS}}\end{array}$ & $\begin{array}{l}23.7 ; \\
<0.001\end{array}$ \\
\hline
\end{tabular}

First mark explore concentration (mean \pm standard error of mean) of macroelements and fibre fractions calculated by one-way ANOVA. Using Tukey post-hoc comparison test, species with the same letter were not significantly different. Second mark explore differences of elements concentrations between two reserves tested by two-sample T-test significant results have: * if $p \leq$ $0.05, * *$ if $p \leq 0.01$ and $* * *$ if $p \leq 0.001$. 


\begin{tabular}{|c|c|c|c|c|c|c|}
\hline & $\begin{array}{l}\text { Derby } \\
\text { eland }\end{array}$ & $\begin{array}{l}\text { Common } \\
\text { eland }\end{array}$ & $\begin{array}{l}\text { Roan } \\
\text { antelope }\end{array}$ & Buffalo & Zebra & $\begin{array}{l}\text { F; p- } \\
\text { value }\end{array}$ \\
\hline FATHALA & $n=36$ & $n=10$ & $n=17$ & $n=7$ & $n=14$ & \\
\hline Nitrogen $\mathrm{g} / \mathrm{kg}$ & $\begin{array}{l}25.6 \pm \\
0.88^{b}\end{array}$ & $\begin{array}{l}25.18 \pm \\
2.08^{b}\end{array}$ & $\begin{array}{l}18.31 \pm 1.47 \\
\mathrm{a}\end{array}$ & $\begin{array}{l}18.03 \pm \\
0.69^{a}\end{array}$ & $\begin{array}{l}15.33^{ \pm} \\
0.82^{a}\end{array}$ & $\begin{array}{l}14.5 \\
<0.001\end{array}$ \\
\hline $\begin{array}{l}\text { Phosphorus } \\
\text { g/kg }\end{array}$ & $\begin{array}{l}5.5 \pm 0.47 \\
b\end{array}$ & $\begin{array}{l}5.90 \pm 0.77 \\
b\end{array}$ & $\begin{array}{l}2.99 \pm 0.32 \\
a\end{array}$ & $\begin{array}{l}2.61 \pm 0.34 \\
\mathrm{a}\end{array}$ & $\begin{array}{l}4.10 \pm 0.60 \\
\mathrm{ab}\end{array}$ & $\begin{array}{l}5.43 \\
<0.001\end{array}$ \\
\hline $\begin{array}{l}\text { Potassium } \\
\mathrm{g} / \mathrm{kg}\end{array}$ & $\begin{array}{l}7.6 \pm 0.43 \\
a b\end{array}$ & $\begin{array}{l}7.60 \pm 1.30 \\
a b\end{array}$ & $\begin{array}{l}5.54 \pm 0.43 \\
a\end{array}$ & $\begin{array}{l}8.11 \pm 0.65 \\
a b\end{array}$ & $\begin{array}{l}9.74 \pm 0.77 \\
b\end{array}$ & $\begin{array}{l}4.88 \\
0.0014\end{array}$ \\
\hline Calcium g/kg & $\begin{array}{l}23.2 \pm \\
1.77 \mathrm{bc}\end{array}$ & $\begin{array}{l}26.57 \pm \\
3.40^{c}\end{array}$ & $\begin{array}{l}10.46 \pm 1.13 \\
\mathrm{a}\end{array}$ & $\begin{array}{l}12.80 \pm \\
0.52 \mathrm{ab}\end{array}$ & $\begin{array}{l}12.66 \pm \\
2.90^{a}\end{array}$ & $\begin{array}{l}9.32 \\
<0.001\end{array}$ \\
\hline $\begin{array}{l}\text { Magnesium } \\
\mathrm{g} / \mathrm{kg}\end{array}$ & $\begin{array}{l}5.7 \pm 0.47 \\
a b\end{array}$ & $\begin{array}{l}7.75 \pm 1.37 \\
b\end{array}$ & $\begin{array}{l}4.63 \pm 0.54 \\
a\end{array}$ & $\begin{array}{l}5.53 \pm 0.40 \\
\text { ab }\end{array}$ & $\begin{array}{l}5.00 \pm 0.29 \\
a b\end{array}$ & $\begin{array}{l}2.43 \\
0.054\end{array}$ \\
\hline Sulphur mg/g & $\begin{array}{l}2.33^{ \pm} \\
0.17^{c}\end{array}$ & $\begin{array}{l}2.14 \pm 0.14 \\
\text { bc }\end{array}$ & $\begin{array}{l}1.84 \pm 0.11 \\
b\end{array}$ & $\begin{array}{l}1.56 \pm 0.05 \\
\mathrm{ab}\end{array}$ & $\begin{array}{l}1.22 \pm 0.05 \\
\mathrm{a}\end{array}$ & $\begin{array}{l}13.1 \\
<0.001\end{array}$ \\
\hline NDF g/kg & $\begin{array}{l}575 \pm 13.7 \\
b\end{array}$ & $433 \pm 55.5^{a}$ & $\begin{array}{l}525 \pm 43.9 \\
a b\end{array}$ & $\begin{array}{l}579 \pm 19.5 \\
a b\end{array}$ & $\begin{array}{l}622 \pm 18.3 \\
b\end{array}$ & $\begin{array}{l}4.32 ; \\
0.003\end{array}$ \\
\hline ADF g/kg & $\begin{array}{l}574 \pm 19.1 \\
\text { b }\end{array}$ & $\begin{array}{l}459 \pm 67.4 \\
a b\end{array}$ & $454 \pm 40.8^{a}$ & $\begin{array}{l}505 \pm 31.7 \\
\mathrm{ab}\end{array}$ & $\begin{array}{l}479 \pm 28.6 \\
\text { ab }\end{array}$ & $\begin{array}{l}3.11 ; \\
0.020\end{array}$ \\
\hline Cellulose & $\begin{array}{l}219 \pm 9.82 \\
a b\end{array}$ & $154 \pm 15.2^{a}$ & $\begin{array}{l}230 \pm 24.6 \\
a b\end{array}$ & $\begin{array}{l}266 \pm 17.1 \\
b c\end{array}$ & $\begin{array}{l}334 \pm 17.9 \\
c\end{array}$ & $\begin{array}{l}11.4 \\
<0.001\end{array}$ \\
\hline Hemicellulose & $\begin{array}{l}55.5 \pm \\
6.84^{a}\end{array}$ & $36.9 \pm 9.6^{a}$ & $71 \pm 9.9^{a}$ & $74 \pm 15.6^{a}$ & $\begin{array}{l}143 \pm 15.5 \\
b\end{array}$ & $\begin{array}{l}12.3 \\
<0.001\end{array}$ \\
\hline Lignin $\mathrm{g} / \mathrm{kg}$ & $\begin{array}{l}355 \pm 14.4 \\
c\end{array}$ & $\begin{array}{l}305 \pm 54.1 \\
b c\end{array}$ & $\begin{array}{l}224 \pm 18.8 \\
a b\end{array}$ & $\begin{array}{l}239 \pm 15.2 \\
a b\end{array}$ & $\begin{array}{l}145 \pm 12.2 \\
a\end{array}$ & $\begin{array}{l}15.9 \\
<0.001\end{array}$ \\
\hline $\begin{array}{l}\text { Lignin } \\
\text { proportion \% }\end{array}$ & $\begin{array}{l}61.6 \pm 1.4 \\
c\end{array}$ & $63.3 \pm 2.8^{c}$ & $52.0 \pm 2.4^{b}$ & $47.3 \pm 0.6^{b}$ & $29.7 \pm 1.2^{a}$ & $\begin{array}{l}47.3 \\
<0.001\end{array}$ \\
\hline Ca:P & $4.25 \pm 0.2^{\mathrm{a}}$ & $4.58 \pm 0.2^{\mathrm{a}}$ & $4.02 \pm 0.4^{\mathrm{ab}}$ & $5.17 \pm 0.7^{a}$ & $\begin{array}{l}2.75 \pm \\
0.34^{\mathrm{b}}\end{array}$ & $\begin{array}{l}5.76 \\
<0.001\end{array}$ \\
\hline \multicolumn{7}{|c|}{$\begin{array}{l}\text { First mark explore concentration (mean } \pm \text { standard error of mean) of macroelements and fibre } \\
\text { fractions calculated by one-way ANOVA. Using Tukey post-hoc comparison test, species with the } \\
\text { same letter were not significantly different. Second mark explore differences of elements } \\
\text { concentrations between two reserves tested by two-sample T-test significant results have: } * \text { if } p \leq \\
0.05, * \star \text { if } p \leq 0.01 \text { and } * \star \star \text { if } p \leq 0.001 \text {. }\end{array}$} \\
\hline
\end{tabular}


Regarding the inter - species comparisons, the diet quality indicated by the concentration of nitrogen was higher in Derby and common elands in comparison to zebra and buffaloes at both sites, while roan antelope was similar to both eland species in the semi-arid and similar to zebra and buffaloes in the subhumid savanna. Hemicellulose, the most digestible fibre fraction in the diet, followed the opposite patterns, being the highest in zebra and the lowest in Derby elands at both sites, conversely to the proportion to indigestible fibres. Phosphorus concentrations in diets did not differ between species in the semi-arid savanna, whereas in the sub humid savanna Derby eland and Common eland showed the highest values. Lignin which is the indicator of browse plants in herbivore's diet, its proportion and calcium were the highest in Derby and common elands at both sites, significantly different from the other species (Table 1).

Microelements reflect the mineral background of the environment and animal physiological state, rather than direct indicators of herbivores diet quality. There was no consistent pattern in response to the savanna type, neither differences among species (Table 2). In the semi-arid savanna, the concentration of $\mathrm{Zn}$ was the highest in buffalo and concentration of Se was the highest in buffalo and roan antelope, while at the sub-humid site concentration of those microelements were highest in browsers, i.e. $\mathrm{Zn}$ in Common eland and Se in Derby eland. Highest concentrations among microminerals were recorded for $\mathrm{Mn}$. 
Table 2

Concentrations (mean \pm SE) of microelements in faeces of large herbivores in Bandia and Fathala reserves.

\begin{tabular}{|c|c|c|c|c|c|c|}
\hline & Derby eland & $\begin{array}{l}\text { Common } \\
\text { eland }\end{array}$ & $\begin{array}{l}\text { Roan } \\
\text { antelope }\end{array}$ & Buffalo & Zebra & F; p-value \\
\hline BANDIA & $n=15$ & $n=11$ & $n=13$ & $n=8$ & $n=15$ & \\
\hline $\mathrm{Mn} \mu \mathrm{g} / \mathrm{g}$ & $\begin{array}{l}162.7 \pm \\
16.94^{\mathrm{ab} / \mathrm{NS}}\end{array}$ & $\begin{array}{l}135 \pm \\
9.81^{\mathrm{a} / \mathrm{NS}}\end{array}$ & $\begin{array}{l}84.56 \pm \\
3.98^{\mathrm{ac} / \star \star \star}\end{array}$ & $\begin{array}{l}100,4 \pm \\
10,35^{\mathrm{ac} / \star \star \star}\end{array}$ & $\begin{array}{l}117.4 \pm \\
13.62^{\mathrm{a} / \mathrm{NS}}\end{array}$ & $\begin{array}{l}5.90 \\
0.001\end{array}$ \\
\hline $\mathrm{Fe} \mathrm{mg} / \mathrm{g}$ & $\begin{array}{l}1.89 \pm \\
0.14^{\mathrm{NS} / \star \star \star}\end{array}$ & $\begin{array}{l}1.79 \pm 0.28 \\
\mathrm{NS} / \mathrm{NS}\end{array}$ & $\begin{array}{l}1.63 \pm 0.1 \\
\text { NS/NS }\end{array}$ & $\begin{array}{l}2,38 \pm 0,4 \\
\text { NS/NS }\end{array}$ & $\begin{array}{l}1.79 \pm 0.2 \\
\text { NS/NS }\end{array}$ & $\begin{array}{l}1.39 \\
0.251\end{array}$ \\
\hline Co $\mu \mathrm{g} / \mathrm{g}$ & $\begin{array}{l}0.84 \pm 0.06 \\
\text { NS/NS }\end{array}$ & $\begin{array}{l}0.82 \pm 0.11 \\
\mathrm{NS} / \star \star \star \star\end{array}$ & $\begin{array}{l}0.72 \pm 0.06 \\
\text { NS/NS }\end{array}$ & $\begin{array}{l}0,93 \pm 0,10 \\
\mathrm{NS} /{ }^{*}\end{array}$ & $\begin{array}{l}0.85 \pm 0.12 \\
\text { NS/NS }\end{array}$ & $\begin{array}{l}0.56 \\
0.696\end{array}$ \\
\hline $\mathrm{Cu} \mu \mathrm{g} / \mathrm{g}$ & $\begin{array}{l}13.41 \pm \\
1.25^{\mathrm{a}^{/ *}}\end{array}$ & $\begin{array}{l}17.4 \pm \\
2.84^{\mathrm{ab} / \mathrm{NS}}\end{array}$ & $\begin{array}{l}15.23 \pm \\
1.68^{\mathrm{a} / \mathrm{NS}}\end{array}$ & $\begin{array}{l}8,31 \pm \\
0,63^{\mathrm{ac} / \mathrm{NS}}\end{array}$ & $\begin{array}{l}12.36 \pm \\
1.64^{\mathrm{a} / \mathrm{NS}}\end{array}$ & $\begin{array}{l}2.81 \\
0.034\end{array}$ \\
\hline $\mathrm{Zn} \mu \mathrm{g} / \mathrm{g}$ & $\begin{array}{l}63.74 \pm \\
3.44^{\text {ac/NS }}\end{array}$ & $\begin{array}{l}66.9 \pm \\
6.77^{\mathrm{a}^{/ *}}\end{array}$ & $\begin{array}{l}67.47 \pm \\
3.22^{\mathrm{a} / \star \star \star}\end{array}$ & $\begin{array}{l}85,48 \pm \\
8,08^{\mathrm{ab} / \star \star}\end{array}$ & $\begin{array}{l}60.40 \pm \\
2.78^{\text {ac/NS }}\end{array}$ & $\begin{array}{l}3.50 \\
0.013\end{array}$ \\
\hline $\mathrm{Se} \mu \mathrm{g} / \mathrm{g}$ & $\begin{array}{l}0.46 \pm \\
0.036^{\mathrm{ac} / \mathrm{NS}}\end{array}$ & $\begin{array}{l}0.71 \pm \\
0.07^{\mathrm{a} / \star \star}\end{array}$ & $\begin{array}{l}0.87 \pm \\
0.10^{\mathrm{ab} / \star \star \star}\end{array}$ & $\begin{array}{l}0,9 \pm \\
0,09^{\mathrm{ab} / \star \star \star}\end{array}$ & $\begin{array}{l}0.72 \pm \\
0.07^{\mathrm{a} / \star \star \star}\end{array}$ & $\begin{array}{l}5.72 \\
0.001\end{array}$ \\
\hline $\mathrm{Mo} \mu \mathrm{g} / \mathrm{g}$ & $\begin{array}{l}0.94 \pm \\
0.06^{\mathrm{b} / \mathrm{NS}}\end{array}$ & $\begin{array}{l}1.66 \pm \\
0.23^{\mathrm{ab} / \mathrm{NS}}\end{array}$ & $\begin{array}{l}2.36 \pm \\
0.26^{\mathrm{a} / \star \star \star}\end{array}$ & $\begin{array}{l}2,33 \pm \\
0,27^{a / \star \star \star}\end{array}$ & $\begin{array}{l}1.82 \pm \\
0.18^{\mathrm{a} / \star \star \star}\end{array}$ & $\begin{array}{l}8.96 ; \\
<0.001\end{array}$ \\
\hline FATHALA & $n=14$ & $n=10$ & $n=15$ & $n=6$ & $n=14$ & \\
\hline $\mathrm{Mn} \mu \mathrm{g} / \mathrm{g}$ & $\begin{array}{l}178.1 \pm \\
16.4^{\text {ac }}\end{array}$ & $\begin{array}{l}157.9 \pm \\
11.04^{a}\end{array}$ & $\begin{array}{l}209.7 \pm \\
13.96^{\mathrm{ac}}\end{array}$ & $\begin{array}{l}197.4 \pm \\
13.75^{a}\end{array}$ & $\begin{array}{l}128.5 \pm \\
6.91^{\mathrm{ab}}\end{array}$ & $\begin{array}{l}6.15 ; \\
<0.001\end{array}$ \\
\hline $\mathrm{Fe} \mathrm{mg} / \mathrm{g}$ & $0.94 \pm 0.11^{\mathrm{ac}}$ & $\begin{array}{l}2.02 \pm \\
0.33^{\mathrm{ab}}\end{array}$ & $1.38 \pm 0.35^{\mathrm{a}}$ & $1.47 \pm 0.1^{\mathrm{a}}$ & $1.47 \pm 0.17^{a}$ & $\begin{array}{l}2.10 \\
0.095\end{array}$ \\
\hline Co $\mu \mathrm{g} / \mathrm{g}$ & $\begin{array}{l}1.00 \pm 0.12 \\
\text { NS }\end{array}$ & $\begin{array}{l}1.6 \pm 0.17 \\
\text { NS }\end{array}$ & $\begin{array}{l}1.02 \pm 0.28 \\
\text { NS }\end{array}$ & $\begin{array}{l}1.84 \pm 0.54 \\
\text { NS }\end{array}$ & $\begin{array}{l}1.33 \pm 0.24 \\
\text { NS }\end{array}$ & $\begin{array}{l}1.53 \\
0.208\end{array}$ \\
\hline $\mathrm{Cu} \mu \mathrm{g} / \mathrm{g}$ & $\begin{array}{l}18.18 \pm \\
1.74^{\mathrm{bc}}\end{array}$ & $22.08 \pm 2.3^{c}$ & $\begin{array}{l}12.75 \pm \\
1.33^{\mathrm{ab}}\end{array}$ & $8.82 \pm 0.24^{\mathrm{a}}$ & $12.3 \pm 0.84^{\mathrm{a}}$ & $\begin{array}{l}8.24 ; \\
<0.001\end{array}$ \\
\hline $\mathrm{Zn} \mu \mathrm{g} / \mathrm{g}$ & $\begin{array}{l}66.57 \pm \\
4.86^{\mathrm{ab}}\end{array}$ & $\begin{array}{l}90.22 \pm \\
8.42^{b}\end{array}$ & $\begin{array}{l}50.54 \pm \\
3.04{ }^{a}\end{array}$ & $\begin{array}{l}51.57 \pm 2.62 \\
\text { a }\end{array}$ & $64.7 \pm 7.86^{a}$ & $\begin{array}{l}5.57 \\
0.0008\end{array}$ \\
\hline
\end{tabular}

First mark explore concentration (mean \pm standard error of mean) of microelements calculated by one-way ANOVA. Using Tukey post-hoc comparison test, species with the same letter were not significantly different. Second mark explore differences of elements concentrations between two reserves tested by two-sample T-test significant results have: * if $p \leq 0.05$,

$\star \star$ if $p \leq 0.01$ and $* \star \star$ if $p \leq 0.001$. 


\begin{tabular}{|c|c|c|c|c|c|c|}
\hline & Derby eland & $\begin{array}{l}\text { Common } \\
\text { eland }\end{array}$ & $\begin{array}{l}\text { Roan } \\
\text { antelope }\end{array}$ & Buffalo & Zebra & F; p-value \\
\hline $\mathrm{Se} \mu \mathrm{g} / \mathrm{g}$ & $0.61 \pm 0.07^{c}$ & $\begin{array}{l}0.45 \pm \\
0.08^{\mathrm{bcd}}\end{array}$ & $\begin{array}{l}0.33 \pm \\
0.02^{\mathrm{ab}}\end{array}$ & $\begin{array}{l}0.26 \pm \\
0.02^{\text {ad }}\end{array}$ & $0.17 \pm 0.02^{\mathrm{a}}$ & $\begin{array}{l}12.66 \\
<0.001\end{array}$ \\
\hline Mo $\mu \mathrm{g} / \mathrm{g}$ & $1.03 \pm 0.17^{a}$ & $\begin{array}{l}1.69 \pm \\
0.38^{\mathrm{ab}}\end{array}$ & $\begin{array}{l}0.65 \pm \\
0.13^{\mathrm{ac}}\end{array}$ & $0.40 \pm 0.02^{\mathrm{ac}}$ & $0.94 \pm 0.12^{\mathrm{a}}$ & $\begin{array}{l}4.39 \\
0.004\end{array}$ \\
\hline \multicolumn{7}{|c|}{$\begin{array}{l}\text { First mark explore concentration (mean } \pm \text { standard error of mean) of microelements calculated by } \\
\text { one-way ANOVA. Using Tukey post-hoc comparison test, species with the same letter were not } \\
\text { significantly different. Second mark explore differences of elements concentrations between two } \\
\text { reserves tested by two-sample T-test significant results have: * if } p \leq 0.05 \text {, }\end{array}$} \\
\hline \multicolumn{7}{|c|}{$\star *$ if $p \leq 0.01$ and $* * *$ if $p \leq 0.001$. } \\
\hline
\end{tabular}

In terms of nutrient landscape pointing out the mutual relationships among nutrients, the multivariate RDA analysis showed significant effects of herbivore species and seasons at both sites; on first axis $\mathrm{F}=$ 2.4, $P=0.001$, on all axes $F=10.4, P=0.001$ in the semi-arid savanna, and on first axis $F=2.7, P=0.001$, on all axes $F=7.1, P=0.001$ in the sub-humid savanna.

At semi-arid site, in the Bandia reserve, the first axis of the RDA explained $20.5 \%$, the second axis $18.1 \%$, the third axis $6.7 \%$, and all four axes together show $50 \%$ of the data variability. The first $(x)$ axis represented the content of $\mathrm{N}, \mathrm{Mg}, \mathrm{P}, \mathrm{S}$, and Cu which were positively and negatively related to the wet and dry seasons, respectively. The second axis represented the content of lignin, $\mathrm{Ca}$ and $\mathrm{Mn}$ with positive relation of Derby and common elands there on one side, and represented hemicellulose, $\mathrm{K}$, and Mo to which roan and buffalo were related closer (Fig. 1a).

At sub-humid site, in the Fathala reserve, the first axis of the RDA explained $24.3 \%$, the second axis $11.1 \%$, the third axis $5.4 \%$, and all four axes together show $44 \%$ of the data variability. The first ( $x$ ) axis predominantly represented the content of $\mathrm{Ca}, \mathrm{P}, \mathrm{Mg}$ together with $\mathrm{Cu}, \mathrm{Zn}$, and Mo which was positively related to wet season and Common eland on one side, and hemicellulose related to buffalo on the other side. The second axis represented mostly lignin and $\mathrm{Mn}$, which were more positively related to Derby eland. On another side second axis also represented $\mathrm{K}$ and Co which were not closely related to any animal species (Fig. 1b). Zebra as a pure grazer stands a side of all other species and elements in both reserves, and is in perfect opposite position to pure browser Derby eland.

\section{Discussion}

Our findings suggest that the mixed feeders and grazers were resilient to the change of habitat by maintaining the level of diet quality in both, sub-humid and semi-arid savanna ecosystems, even if those substantially differ in the vegetation structure and plant species composition. Few differences found in zebra and buffalo in the sub-humid ecosystem with higher NDF and ADF fibre content indicated a higher proportion of fresh grass biomass in grazers' diet. Similar proportion of lignin in zebra's diet in semi-arid 
and sub-humid conditions indicated that zebra did not incorporate any higher proportion of browse to its diet despite grass limited environment, similarly as reported by [25]. Such feeding strategy put other grazing herbivores, here namely roan antelope and buffalo, under a strong competition. Higher proportions of lignin in the diet of roan, and buffalos in the sub-humid savanna suggest that they include browse in their diet as elsewhere, specifically for buffalo see [26], [27], [28]. It seems contradictory as buffalos are primarily grazers and roan antelopes switch their diet to browsing especially during dry seasons when resources are scarce, switching from strict grazing ( $>95 \%$ grass) to mixed feeding ( $<50 \%$ grass) [19], whereas in our study, we expect there were more resources in the sub-humid savanna. Our results suggest that the vegetation structure of the sub-humid savanna that is composed by denser woody plants canopy and tall grasses (Andropogon gayanus, Schizachyrium sanguineum) and herbs in the undergrowth [29] does not offer for grazers adequate grass resources, i.e. appropriate leaf-stem ratio [30]. Roan antelope are sensitive to habitat change and competition from sympatric grazers and are susceptible to nutritional deficiencies [31]. Therefore, facing the spatial limitation, i.e. no option to migrate, and competition from other bulky feeders, i.e., zebras, the species that are physiologically able to switch from grazing to browsing on trees, shrubs and herbs [32], do so and maintain their diet quality.

Browsers are considered most resilient, not changing the diet, nor the use of space during drought events because trees are more resistant to drought and provide forage resources more or less continuously [3; 13]. Derby elands browse on a wide variety of woody species and are not particularly selective [33; 15], therefore we expected the animals to find adequate food resources in both environments, despite different vegetation. Their diet, however, differed in quality, specifically the animals' diet was lower in macroelements and fibres in the semi-arid savanna in comparison to the sub-humid one. The levels of nutrients, however, did not fall under critical limits which could lead animals to nutritional deficiency. For large African herbivores, suggested critical faecal nitrogen concentration lies within the range $13-16 \mathrm{~g} / \mathrm{kg}$ of dry matter (1.3-1.6\% of dry matter) and critical faecal phosphorus concentration is estimated to 2 $\mathrm{g} / \mathrm{kg}(0.2 \%$ of dry matter) [34;35]. Especially pronounced difference in the diet quality parameters between the two sites for browsers, i.e. for Derby eland, but also for common eland, was found in the content of lignin and its proportion as indigestible component in the diet. The sub-humid savanna offers broad-leaved tree species, for instance Terminalia spp., Combretum spp., Saba senegalensis [36], these species thus provide more biomass, while they also contain more lignin, tannins, and/or other secondary metabolites which simultaneously increase nitrogen content irrelevant as nutritive element due to its nonprotein origin. On the other hand, the semi-arid savanna vegetation is composed dominantly by Acacia spp. with more narrow leaves, less biomass, with less antinutritive compounds. The consumption of Acacia spp. by browsing animals is indirectly confirmed by very high concentration of $\mathrm{Ca}$ and strongly biased Ca:P ratio, especially in Derby eland diet, as legume, including Acacia spp., and other tree and shrub species alike are rich source of $\mathrm{Ca}[37 ; 38]$. Elands having $\mathrm{Ca}$ rich and $\mathrm{P}$ poor diet are likely unable to absorb $\mathrm{Ca}$ from the diet and then Ca leave the body without utilization [39].

There was no specific consistent pattern in the microelement concentrations in relation to feeding type, species, or savanna type. In tropical regions, soils often have limiting supplies of trace elements [40]. This environmental background translates into plant food and as a consequence, domestic ungulates in 
tropical regions feeding on natural resources were reported as mineral deficient [41]. Data from wild large savanna herbivores on microelements are virtually inexistent. In our study, we have not found any low values of microelements which would indicate any deficiency. Nevertheless, Mn concentration at all species at both localities are very high in comparison to, for instance, $4 \mu \mathrm{g} / \mathrm{kg}$ maintenance requirements of ewes [42]. High concentrations of $\mathrm{Mn}$ are even sharply increased at roan antelopes and buffaloes in the sub-humid savanna. On the other hand, high Mn concentrations can be toxic, leading to depressed iron status and hematologic changes or damage of rumen microbial flora. Despite the fact, that $\mathrm{Mn}$ is one of the least toxic of the essential elements [43] concentrations of $\mathrm{Mn}$, as well as of other microelements, and their sources from environment should be investigated further.

We must pay a close attention to the supplementary feeding and its role in the diet quality during the hot dry season when the supplement is supplied to animals. The food supplement is composed by Acacia albida (synonymous to Faidherbia albida) pods and peanut hay (Arachis hypogea). Both are legume species, rich in nitrogen, phosphorus, and calcium $[44 ; 45 ; 46]$ and supplied to maintain the animals' fitness during the most critical period of scarce natural resources, especially in regard to conservation program of critically endangered western subspecies of Derby eland. The supplement may change the interplay of all diet components and prevents a meaningful comparison to free ranging wildlife. For instance, faecal nitrogen and phosphorus concentrations in the animals' diet in our study were higher at both sites and for all species in comparison to the same and related species during the dry season in Kruger national park [47]. Similarly, the Ca:P ratio in diet of browsing species, i.e. both browsers and intermediate, was higher in the semi-arid savanna reserve where the supplementing management is more regular and intensive in comparison to the reserve in sub-humid area. The multivariate analysis in the sub-humid savanna showed the importance of ongoing season from the wet season on one side, through dry season with low explanatory value, to the hot dry on the other side, and this pattern suggests that a supplementary feeding was complementary only, without any substantial impact on natural dietary associations. Two species of mixed feeders, roan antelope and common eland, showed opposite association to nutrients and seasons, confirming thus their distinct features, thus incomparability despite their 'mixed feeders' designation. The position of roan antelope shows its similarity to buffalo and previous consideration of buffalo's partial browsing. Derby eland related especially to lignin and nitrogen, and zebra appear independent on the season, each species on the opposite side. In contrast, the multivariate analysis of nutrients, species and seasons in the semi-arid savanna revealed the importance of wet and dry seasons, while the hot dry season appeared to be of low importance for the nutrients in the animal diet. We associate this to the effect of supplementary feeding which changes the relationships of nutrients in animal diets and similarities among animals. Under these conditions, common eland diet was more similar to Derby eland's diet, and their relation to Ca suggest that they benefit from supplementary feeding, alike buffalo was more similar to zebra, all these species independent of season. Diet of roan antelope was more distinct from other species and remained on the axis of seasons.

\section{Conclusion}


Overall, our results showed that despite differences in aggregate rainfall and vegetation structure (including plant species composition) between semi-arid and sub-humid savannas at two sites, grazers and mixed feeders maintained the diet quality in most nutrients at similar levels. Our findings suggest that to maintain the level of diet quality, the conditions of different ecosystems with no possibility to leave the area imposed changes in feeding behaviour across feeding types, most pronounced in mixed feeders. Based on proportion of indigestible lignin in faeces, we infer that buffalos and roan antelopes escaped the competition with non-native zebras for grasses in sub-humid savanna where denser canopy and mostly herbs in the undergrowth make grasses a scarce resource, by switching to browse. Therefore, introduction of not native species into native communities may have a big impact on behaviour and may force them to go to the limits of their feeding resilience. In terms of diet quality, the species differing the most between the two ecosystems was Derby eland, the pure browser, showing consistently lower levels of all macroelements and fibres in the semi-arid environment. Therefore, browsers may experience intensifying impacts in terms of diet quality and available nutrients, despite they have been considered not to be affected behaviourally by drier conditions, for instance by drought events, based on assumption that woody plant resources remain available and browsers thus appeared more resilient [3]. From conservation perspective, all feeding guilds may become threatened by changing ecosystems, even if each one by different way, e.g. by increased competition or inadequate nutrient levels if they are forced to change food resources, and a conservation-oriented 'exit-strategy' for species should be conceived to address these threats, including the strengthening the ecological connectivity in savanna landscapes.

\section{Declarations}

\section{DATA AVAILABILITY STATEMENT}

The data that support the findings of this study are available from the corresponding author upon request.

\section{ACKNOWLEDGEMENTS}

We thank Karolína Brandlová and Pavla Jůnková Vymyslická for their assistance with the field sample collection, as well as Derbianus Conservation NGO (www.derbianus.cz) and the managers and staff of Bandia and Fathala reserves in Senegal for facilitating the research. This work was made possible by funding of the Czech University of Life Sciences Prague, Faculty of Tropical AgriSciences, by grant number IGA 20205015, and of the Ministry of Education of the Czech Republic, grant number CZ.02.2.69/0.0/0.0/19_074/0016295.

\section{CONFLICT OF INTEREST}

None declared.

\section{AUTHOR CONTRIBUTION}


PH conceived the study, designed and collected samples in the field. LS processed the samples and conducted laboratory analyses. PH and LS performed statistical analyses and contributed to writing equally.

\section{References}

1. McNaughton SJ, Georgiadis NJ. 1986. Ecology of African grazing and browsing mammals. Annual Review of Ecology and Systematics 17:39-65. DOI: 10.1146/annurev.es.17.110186.000351

2. Kleynhans EJ, Jolles AE, Marjan RE, Olff BH. 2011. Resource partitioning along multiple niche dimensions in differently sized African savanna grazers. Oikos 120: 591-600. DOI: 10.1111/j.16000706.2010.18712.x

3. Abraham JO, Hempson GP, Staver AC. 2019. Drought-response strategies of savanna herbivores. Ecology and Evolution 9:7047-7056. DOI: 10.1002/ece3.5270

4. Prins HHT, Boer de WF, Oeveren van H, Correia A, Mafuca J, Olff H. 2006. Co-existence and niche segregation of three small bovid species in southern Mozambique. African Journal of Ecology 44:186-198. DOI: 10.1111/j.1365-2028.2006.00619.x

5. Kartzinel TR, Chen PA, Coverdale TC, Erickson DL, Kress WJ, Kuzmina ML, Rubenstein DI, Wang W, Pringle RM. 2015. DNA metabarcoding illuminates dietary niche partitioning by African large herbivores. PNAS 26:8019-8024 DOI: 10. 1073/pnas.1503283112

6. McNaughton. 1985. Ecology of a Grazing Ecosystem: The Serengeti. Ecological Monographs 55:259294. DOI: $10.2307 / 1942578$

7. Voeten MM, Prins HHT. 1999. Resource partitioning between sympatric wild and domestic herbivores in the Tarangire region of Tanzania. Oecologia 120:287-294. DOI: 10.1007/s004420050860

8. Lyon B. 2004. The strength of El Niño and the spatial extent of trop-ical drought. Geophysical Research Letters 31:L21204. DOI: 10.1029./2004GL020901

9. Knapp AK, Beier C, Briske DD, Classen AT, Luo Y, Reichstein M, et al. 2008. Consequences of more extreme precipita- tion regimes for terrestrial ecosystems. BioScience, 58(9):811-821. DOI: 10.1641/B580908

10. Hulme M, Doherty R, Ngara TNM, Lister D. 2001. African climate change: 1900 - 2100. Climate Research 17: 145 - 168. DOI: 10.3354/cr017145

11. Thuiller W, Broennimann O, Hughes G, Alkemade JRM, Midgley GF, Corsi F. 2006. Vulnerability of African mammals to anthropogenic climate change under conservative land transformation assumptions. Global Change Biology 12: 424-440. DOI: 10.1111/j.1365-2486.2006.01115.x

12. Augustine DJ. 2010. Response of native ungulates to drought in semi-arid Kenyan rangeland. African Journal of Ecology 48: 1009-1020. DOI: 10.1111/j.1365-2028.2010.01207.x

13. Craigie ID, Baillie JE, Balmford A, Carbone C, Collen B, Green RE, Hutton JM. 2010. Large mammal population declines in Africa's protected areas. Biological Conservation 143: 2221-2228. DOI: 10.1016/j.biocon.2010.06.007 
14. Hejcmanová P, Homolka M, Antonínová M, Hejcman M, Podhájecká V. 2010. Diet composition of western Derby eland (Taurotragus derbianus derbianus) in the dry season in natural and managed habitat in Senegal using faecal analysis. South African Journal of Wildlife Research 40(1):27-34. DOI: $10.3957 / 056.040 .0105$

15. Hejcmanová P, Miřejovská M, Homolka P, Hejcman M. 2019. Drivers of diet selection of critically endangered Western Derby eland during the food shortage period within conservation breeding in Senegal. Scientific reports: 8712. DOI: 10.1038/s41598-019-45035-z

16. Sponheimer M, Lee-Thorp JA, DeRuiter DJ, Smith JM, Van der Merwe J, Grant CC, Ayliffe LK, Robinson TF, Heidelberger C, Marcus W. 2003. Diets of southern African Bovidae: stable isotope evidence. Journal of Mammalogy 84:471-479. DOI: 10.1644/1545-1542

17. Codron D, Lee-Thorp JA, Sponheimer M, Codron J. 2007. Nutritional content of savanna plant foods: implications for browser/grazer models of ungulate diversification. Eur J Wildl Res 53:100-111. DOI: 10.1007/s10344-006-0071-1

18. Mandlate JRLC, Arsenault R, Rodrigues FHG. 2019. Grass greenness and grass height promote the resource partitioning among reintroduced Burchell's zebra and blue wildebeest in southern Mozambique. Austral Ecology 44:648-657. DOI: 10.1111/aec.12708

19. Schuette JR, Leslie DM Jr, Lochmiller RL, Jenks JA. 1998. Diets of heartebeest and roan anelope in Burkina Faso: support of the long-faced hypothesis. Journal of Mammalogy 79: 426-436.

20. Tyowua BT, Agbelusi EA, Orsar JT. 2013. Roan antelope (Hippotragus equinus, Desmarest 1804) food plants and feeding habits in Kainji Lake National Park, Nigeria. Journal of Research in Forestry, Wildlife and Environment 4:22-29.

21. Parrini F, D'Ammando G, Marshal JP. 2019. Seasonal shifts in habitat use and diet by eland confined in a small fenced reserve. African Journal of Ecology 57:394-403. DOI: 10.1111/aje.12618

22. Hejcmanová P, Ortmann S, Stoklasová L, Clauss M. 2020. Digesta passage in common eland (Taurotragus oryx) on a monocot or a dicot diet. Comparative Biochemistry and Physiology, Part A 246. 110720. DOI: $10.1016 /$ j.cbpa.2020.110720

23. AOAC International.1995. Official methods of analysis of AOAC International. Volume 1. 16th edition. Arlington, USA, Association of Analytical Communities. ISBN :0935584544

24. Ter Braak CJF, Šmilauer P. 2012. Canoco reference manual and user's guide: software for ordination, version 5.0. Microcomputer Power, Ithaca, USA, 496 pp.

25. Landman M, Kerley GIH. 2001. Dietary shifts: do grazers become browsers in the Thicket Biome? Koedoe 44: 31-36.

26. Jarman PJ. 1971. Diets of Large Mammals in the Woodlands around Lake Kariba, Rhodesia. Oecologia 8: 157-178.

27. Mloszewski MJ. 1983. The Behavior and Ecology of the African Buffalo. Cambridge University Press,Cambridge. DOI: 10.2307/2405076

28. Gagnon M, Chew AE. 2000. Dietary preferences in extant african bovidae. Journal of Mammalogy 81(2):490-511. DOI: 10.1093/jmammal/81.2.490 
29. Nežerková-Hejcmanová P, Hejcman M, Camara AA, Antonínová M, Pavlů V, Černý T, Bâ AT. 2005. Analysis of herbaceous undergrowth in woody savanna in Fathala Reserve, Delta du Saloum National Park, Senegal. Belgian Journal of Botany 138:119-128.

30. Heitkönig IMA, Owen-Smith N. 1998. Seasonal selection of soil types and grass swards by roan antelope in a South African savanna. African Journal of Ecology 36: 57-70.

31. Wilson DE, Hirst SM. 1977. Ecology and factors limiting roan and sable antelope populations in South Africa. Wildlife Monographs 54: 3-111.

32. Clauss M, Lechner-Doll M, Streich WJ. 2003. Ruminant diversification as an adaptation to the physicomechanical characteristics of forage. A reevaluation of an old debate and a new hypothesis. Oikos 102:253-262. DOI: 10.1034/j.1600-0706.2003.12406.x

33. Hejcmanová P, Vymyslická P, Žáčková M, Hejcman M. 2013. Does supplemental feeding affect behaviour and foraging of critically endangered western giant eland in an ex situ conservation site? African Zoology 48(2):250-258. DOI: 10.3377/044.048.0205

34. Wrench JM. 1997. The use of faecal analyses to predict dietary composition in wild herbivores. M.Sc. thesis. University of Pretoria, Pretoria.

35. Grant CC, Peel MJS, Zambatis N, Van Ryssen JBJ. 2000. Nitrogen and phosphorus concentration in faeces: an indicator of range quality as a practical adjunct to existing range evaluation methods. Afr. J. Range Forage Sci. 17: 81-92. DOI: 10.2989/10220110009485743

36. Žáčková M, Hejcmanová P, Hejcman M. 2013. Does a change in land use affect woody vegetation in sub-humid sudanian savanna in Senegal? Scientia Agriculturae Bohemica, 44:209-2017. DOI: $10.7160 /$ sab.2013.440403

37. Topps JH. 1992. Potential, composition and use of legume shrubs and trees as fodders for livestock in the tropics. Journal of Agricultural Science 118:1-8. DOI: 10.1017/S0021859600067940

38. Tefera S, Mlambo V. 2017. Macro and trace elements of 26 browse species growing in semi-arid grazing lands of Eastern Cape, South Africa. African Journal of Range and Forage Science 34:209217. DOI: 10.2989/10220119.2017.1412354

39. Stoklasová L, Váhala J, Hejcmanová P. 2019. Minerals in the Blood, Hair, and Faeces of the Critically Endangered Western Derby Eland Under Human Care in Two Wildlife Reserves in Senegal. Biological Trace Element Research 195:105-109. DOI: 10.1007/s12011-019-01839-8

40. Hacker JB ed. 1982. Nutritional Limits to Animal Production From Pastures. Slough: Commonwealth Agric. Bur. 548 pp. IBSN: 0851984924

41. McDowell LR, Conrad JH, Ellis GL. 1983. Mineral Deficiencies and Imbalances and Their Diagnosis. Proc. Symp. Herbivore Nutrition in the subtropics and tropics, Pretoria pp.67-88.

42. Zhang H, Nie H, Wang Z, Wang F. 2018. The net iron, manganese, copper, and zinc requirements for maintenance and growth of Dorper x Hu ewe lambs. Italian Journal of Animal Science 17:941-949. DOI: 10.1080/1828051X.2018.1431964

43. Herdt T, Hoff B. 2011. The use of blood analysis to evaluate trace mineral status in Ruminant livestock. Vet Clin Food Anim 27: 255-283. DOI: 10.1016/j.cvfa.2011.02.004 
44. Hassan LG, Umar KJ, Yuguda H. 2007. Nutritional evaluation of Faidherbia albida seeds and pulp as source of feeds for livestock. African Journal of Food, Agriculture, Nutrition and Development, 7(5).

45. Mokgolodi NC, Setshogo MP, Shi LL, Liu YJ, Ma C. 2011. Achieving food and nutritional security through agroforestry: a case of Faidherbia albida in sub-Saharan Africa. Forestry Studies in China 13(2):123-131. DOI: 10.1007/s11632-011-0202-y

46. Khan MT, Khan NA, Bezabih M, Qureshi MS, Rahman A. 2013. The nutritional value of peanut hay (Arachis hypogaea L.) as an alternate forage source for sheep. Tropical Animal Health and Production 45(3):849-853. DOI: 10.1007/s11250-012-0297-8

47. Macandza V, Owen-Smith N, Le Roux E. 2013. Faecal nutritional indicators in relation to the comparative population performance of sable antelope and other grazers. African Journal of Ecology 52:300-307. DOI: 10.1111/aje.12119

\section{Figures}



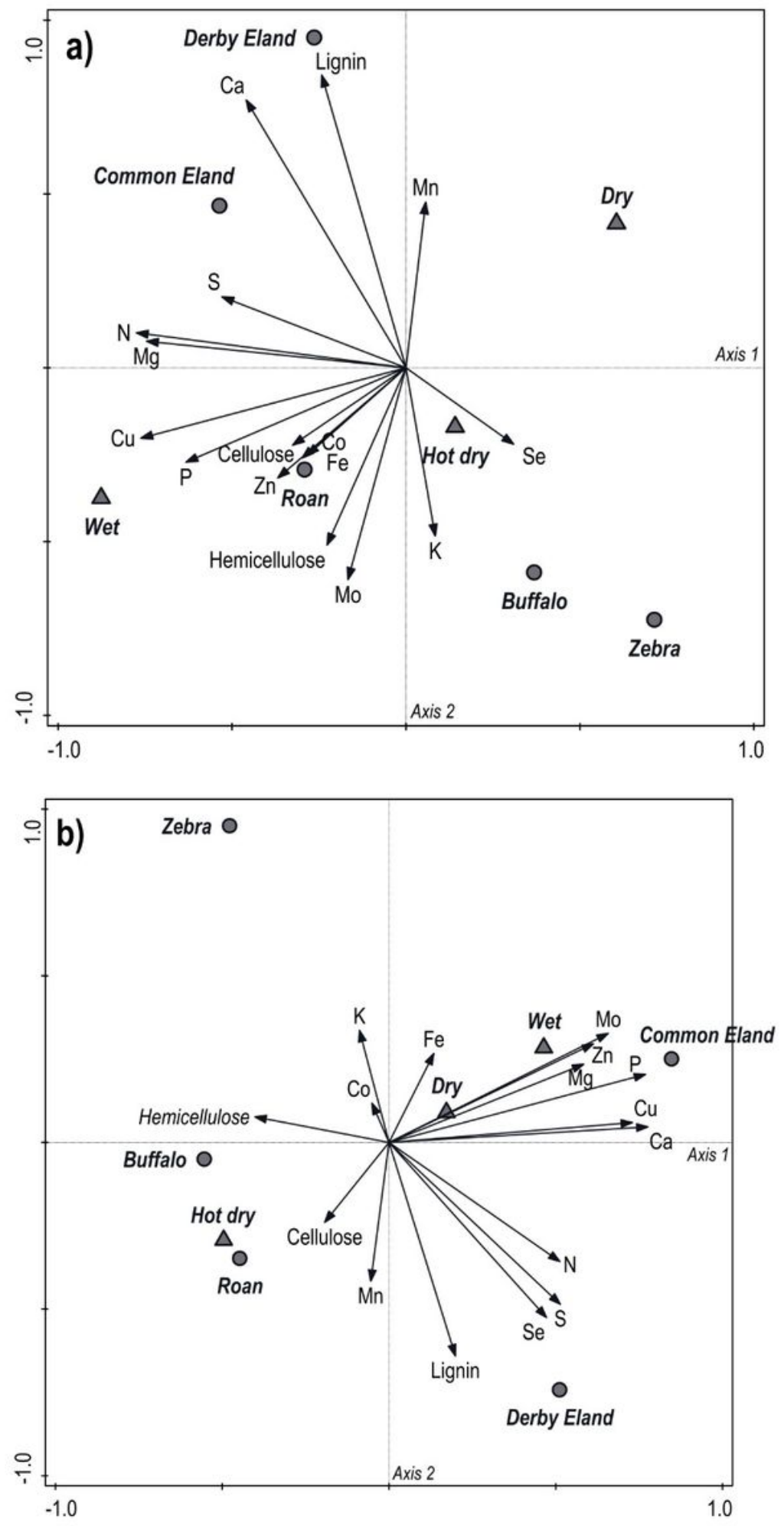

\section{Figure 1}

Constrained redundancy analysis ordination diagram showing mutual relationships of nutrients, i.e. macro-, microelements and fibre fractions, and effects of animal species and seasons in the a) semi-arid savanna in the Bandia reserve, and b) sub-humid savanna in the Fathala reserve. 